\title{
EXPERIENCIAS DOCENTES EN ÁLGEBRA LINEAL CON MAXIMA: PONTE EN EL LUGAR DEL PROFESOR
}

\author{
BEATRIZ ESTRADA \\ Departamento de Matemáticas Fundamentales \\ Facultad de Ciencias, U.N.E.D. \\ Paseo Senda del Rey, 9. 28040 Madrid, Spain. \\ email: bestra@mat.uned.es. \\ Presentado en eXIDO17 (2017) cc) (i) (8)
}

\section{INTRODUCCIÓN}

Desde la aparición del Espacio Europeo de Educación Superior y las nuevas titulaciones de Grados, se recogen en las memorias de estos títulos multitud de competencias relacionadas con las TIC y con la capacidad de comunicación que deben desarrollar los estudiantes. Los docentes, por tanto, debemos promover de algún modo actividades que exijan a los estudiantes desarrollar esas competencias en paralelo al estudio de los contenidos académicos propios de cada asignatura. $\mathrm{Y}$ viceversa, podemos aprovechar el uso de actividades relacionadas con las TIC, como en este caso se hace con el potencial de una herramienta de cálculo simbólico, para profundizar en los conceptos abordándolos desde puntos de vista distintos al usual, desde un punto de vista más orientado a la investigación y la experimentación.

En este sentido, se expone una experiencia docente en la que se ha perseguido este propósito. En concreto, se trata de actividades que implican el uso del programa de cálculo simbólico Maxima en el contexto de estudio del Álgebra Lineal.

\section{Contextualización De la experiencia}

La experiencia ha tenido origen en un aula de apoyo a estudiantes de Álgebra Lineal II, asignatura de primer curso del grado en Matemáticas de la UNED, y surgió ante el asombro que me manifestó un estudiante cuando resolvíamos un ejercicio: ¿cómo se le ha ocurrido ese ejercicio? Mientras explicaba cómo hace el profesor para elaborar el enunciado de un ejercicio tuvo lugar una experiencia muy enriquecedora para todos. 
El grado en Matemáticas incluye una asignatura denominada Herramientas Informáticas para las Matemáticas, en la que aprenden a utilizar programas como: Maxima y R, y el editor profesional de escritura matemática $\mathrm{LT}_{\mathrm{E}} \mathrm{X}$. Aunque esto se hace en cursos posteriores, en las signaturas Álgebra Lineal I y II, les animamos desde el principio a que utilicen algún programa de cálculo simbólico para que se vayan familiarizando.

Aprovechando la idea de explicar cómo el profesor confecciona el enunciado de un ejercicio ayudándose de un programa de cálculo, les propuse una sesión organizada de trabajo en la que ellos mismos participaran en la elaboración de enunciados utilizando Maxima.

La infraestructura que utilizamos fue un aula con pizarra digital donde todos los estudiantes pudiesen ver los resultados de los cómputos.

\section{Experimentando CON El CÁlCUlo matricial}

En la propuesta de trabajo con los estudiantes se pretendía experimentar con dos tipos de problemas básicos en Álgebra Lineal:

- Relaciones de semejanza entre matrices: dentro del estudio de las aplicaciones lineales que son endomorfismos y obtención de sus formas canónicas.

- Relaciones de congruencia entre matrices simétricas: dentro del estudio de las formas bilineales y cuadráticas reales.

Distinguimos dos tipos de ejercicios en este contexto de estudio

\section{(1) Ejercicios directos:}

"Dada una matriz cuadrada (matriz de una aplicación lineal) determinar: sus autovalores y autovectores, la forma canónica de Jordan y la base asociada, decidir si es o no diagonalizable"

"Dada una matriz cuadrada real y simétrica (matriz de una forma bilineal simétrica o una forma cuadrática real): determinar su signatura por distintos, métodos y una base de vectores conjugados". Congruencia.

(2) Ejercicios inversos: Determinar la matriz de una aplicación lineal o la de una forma cuadrática que cumple una serie de condiciones geométricas dadas.

La propuesta de trabajo: elaborar enunciados de problemas directos e inversos como los expuestos anteriormente, trabajando en grupo en el aula de forma guiada por el profesor. Se reflexiona en cada paso, profundizando en las propiedades y conceptos que se van utilizando. El profesor guía la sesión y da pistas y ayudas, pero no se avanza sin una propuesta concreta de los estudiantes. 


\section{Ejemplos De algunos De los EnUNCiAdos Elaborados}

\section{Un ejemplo de problema directo}

Objetivo. Determinar un enunciado del tipo: Dada una matriz $A$ de orden 3 correspondiente a un endomorfismo, determine su forma canónica de Jordan y la base de Jordan asociada.

Paso 1: ¿Cómo queremos que sea la forma canónica de Jordan de A? Debate y propuesta: que tenga un autovalor doble y uno simple.

$$
M_{\mathcal{B}}=\left(\begin{array}{ccc}
2 & 0 & 0 \\
0 & 2 & 0 \\
0 & 0 & 3
\end{array}\right) \quad \text { o bien } \quad M_{\mathcal{B}}=\left(\begin{array}{lll}
2 & 0 & 0 \\
1 & 2 & 0 \\
0 & 0 & 3
\end{array}\right)
$$

Tenemos dos opciones según queramos que sea o no diagonalizable.

Paso 2: ¿Cómo determinar una matriz cuya forma de Jordan sea esa? Debate sobre relaciones de semejanza que justifican el siguiente paso.

Paso 3: Se elige una base que será la de Jordan $\mathcal{B}$ y se considera la matriz $P$ cuyas columnas son los vectores de dicha base. El profesor hace el cambio de base $\mathcal{B}$ a la canónica $\mathcal{C}$ con Maxima

$$
P \cdot M_{\mathcal{B}} \cdot P^{-1}=M_{\mathcal{C}}
$$

y se van debatiendo sobre los resultados.

$$
\begin{aligned}
& \left(\begin{array}{ccc}
2 & 1 & 3 \\
1 & 0 & -1 \\
1 & -1 & 1
\end{array}\right)\left(\begin{array}{lll}
2 & 0 & 0 \\
0 & 2 & 0 \\
0 & 0 & 3
\end{array}\right)\left(\begin{array}{ccc}
2 & 1 & 3 \\
1 & 0 & -1 \\
1 & -1 & 1
\end{array}\right)^{-1}=\left(\begin{array}{ccc}
\frac{17}{7} & -\frac{9}{7} & \frac{3}{7} \\
-\frac{1}{7} & \frac{17}{7} & -\frac{1}{7} \\
\frac{1}{7} & -\frac{3}{7} & \frac{15}{7}
\end{array}\right) \\
& \left(\begin{array}{ccc}
2 & 1 & 3 \\
1 & 0 & -1 \\
1 & -1 & 1
\end{array}\right)\left(\begin{array}{lll}
2 & 0 & 0 \\
1 & 2 & 0 \\
0 & 0 & 3
\end{array}\right)\left(\begin{array}{ccc}
2 & 1 & 3 \\
1 & 0 & -1 \\
1 & -1 & 1
\end{array}\right)^{-1}=\left(\begin{array}{ccc}
\frac{18}{7} & -\frac{5}{7} & \frac{4}{7} \\
-\frac{1}{7} & \frac{17}{7} & -\frac{1}{7} \\
0 & -1 & 2
\end{array}\right)
\end{aligned}
$$

Tenemos dos opciones: elegimos la segunda, el caso no diagonalizable.

Reflexión: Todos concluyen que en caso tener que resolver ellos el ejercicio les gustaría una matriz con entradas más sencillas. ¿Enteras?, ¿cómo?

Paso 3: Concretamos un objetivo. Determinar $A$ de orden 3 , no triangular que tenga autovalores: 2 (doble) y 3 (simple). No diagonalizable y con entradas enteras. Probamos con distintas matrices de laso $P$ pero no se mejora mucho.

Ayuda: Si $P$ tiene entradas enteras, una condición suficiente para que $P^{-1}$ tenga entradas enteras es $\operatorname{det} P= \pm 1$.

Debate: ¿como conseguir matrices con determinante \pm 1 ? La primera opción que surge es que sean triangulares ya que su determinante se calcula muy fácilmente. Probamos 
una con Maxima:

$$
\left(\begin{array}{ccc}
1 & 1 & 3 \\
0 & 1 & -1 \\
0 & 0 & 1
\end{array}\right)\left(\begin{array}{ccc}
2 & 0 & 0 \\
1 & 2 & 0 \\
0 & 0 & 3
\end{array}\right)\left(\begin{array}{ccc}
1 & 1 & 3 \\
0 & 1 & -1 \\
0 & 0 & 1
\end{array}\right)^{-1}=\left(\begin{array}{ccc}
3 & -1 & -1 \\
1 & 1 & -5 \\
0 & 0 & 3
\end{array}\right)
$$

Paso 4: Nuevo objetivo: Otras opciones para $P$ de modo que no se identifiquen los autovalores de la matriz sin previo cálculo.

Ayuda: que $P$ no sea triangular pero tenga estructura triangular por bloques y sigamos controlando su determinante.

$$
\left(\begin{array}{l|ll}
1 & 1 & 1 \\
\hline 0 & 2 & 1 \\
0 & 1 & 1
\end{array}\right)\left(\begin{array}{lll}
2 & 0 & 0 \\
1 & 2 & 0 \\
0 & 0 & 3
\end{array}\right)\left(\begin{array}{lll}
1 & 1 & 1 \\
0 & 2 & 1 \\
0 & 1 & 1
\end{array}\right)^{-1}=\left(\begin{array}{ccc}
3 & -1 & 1 \\
2 & 1 & 0 \\
1 & -1 & 3
\end{array}\right)
$$

Más opciones: Si $P$ tiene determinante \pm 1 y hacemos permutaciones de sus filas y/o columnas, obtenemos otra matriz regular con determinante \pm 1 . Así, $P^{-1}$ sigue teniendo entradas enteras.

$$
\left(\begin{array}{lll}
1 & 1 & 1 \\
0 & 1 & 1 \\
0 & 2 & 1
\end{array}\right)\left(\begin{array}{lll}
2 & 0 & 0 \\
1 & 2 & 0 \\
0 & 0 & 3
\end{array}\right)\left(\begin{array}{lll}
1 & 1 & 1 \\
0 & 1 & 1 \\
0 & 2 & 1
\end{array}\right)^{-1}=\left(\begin{array}{ccc}
3 & 1 & -1 \\
1 & 3 & -1 \\
2 & 0 & 1
\end{array}\right)
$$

Se obtuvieron dos enunciados:

Ejercicio 1. Determine la forma canónica de Jordan del endomorfismo cuya matriz es

$$
\left(\begin{array}{ccc}
3 & 1 & -1 \\
1 & 3 & -1 \\
2 & 0 & 1
\end{array}\right)
$$

Ejercicio 2. Determine la forma canónica de Jordan del endomorfismo cuya matriz es

$$
\left(\begin{array}{ccc}
3 & -1 & 1 \\
2 & 1 & 0 \\
1 & -1 & 3
\end{array}\right)
$$

En ambos casos sabemos que la solución es la matriz de la que hemos partido:

$$
\left(\begin{array}{lll}
2 & 0 & 0 \\
1 & 2 & 0 \\
0 & 0 & 3
\end{array}\right)
$$

y también tenemos una base de Jordan.

En esta parte se trabajaron:

- Propiedades de la semejanza de matrices.

- Cambios de base.

- Propiedades de la inversa.

- Propiedades de los determinantes. 


\section{Un ejemplo de problema inverso:}

Objetivo. Determinar un enunciado del tipo: Obtenga la matriz en la base canónica de una aplicación lineal $f: \mathbb{K}^{3} \rightarrow \mathbb{K}^{3}$ dadas unas condiciones geométricas.

Punto de partida: utilizar uno de los ejemplos previos, el (4.1).

Trabajo a desarrollar: considerando los vectores de la base de Jordan dados como columnas en $P$, interpretar condiciones geométricas sobre los subespacios vectoriales asociados.

Se consiguieron dos enunciados cuya solución sería la matriz del Ejercicio 1:

Ejercicio 3. Determine la matriz de la aplicación lineal $f: \mathbb{K}^{3} \rightarrow \mathbb{K}^{3}$ que satisface las siguientes condiciones

a) $f(1,1,2)=(2,2,4)$,

b) $f(1,0,0)=(3,1,2)$,

c) El vector $(1,1,1)$ es autovector asociado al autovalor $\lambda=3$.

Ejercicio 4. Determine la matriz de la aplicación lineal $f: \mathbb{K}^{3} \rightarrow \mathbb{K}^{3}$ que satisface las siguientes condiciones

a) El vector $(1,1,2)$ es autovector asociado al autovalor 2 .

b) $f(1,0,0)=(3,1,2)$

c) $\lambda=3$ es autovalor de $f$ y el subespacio propio es la recta de ecuaciones $x=y=z$.

\section{El caso de un ejercicio de la Prueba de Evaluación Continua}

Con ayuda del profesor, se reconstruyó el proceso de elaboración del ejercicio del último examen que habían realizado, una prueba de evaluación continua. El enunciado era el siguiente:

Determine la forma canónica de Jordan de un endomorfismo $f$ de $\mathbb{K}^{5}$ que respecto de una base $\mathcal{B}=\left\{v_{1}, v_{2}, v_{3}, v_{4}, v_{5}\right\}$ cumple las siguientes condiciones:

(a) $f\left(v_{1}\right)=-v_{1}$

(b) $f\left(v_{5}\right)=-v_{1}$

(c) $f\left(v_{2}-v_{3}\right)=v_{3}-v_{2}$

(d) $\operatorname{Ker}(f-2 I d) \neq \operatorname{Ker}(f-2 I d)^{2} \equiv\left\{x_{1}+x_{2}-x_{3}=0, x_{1}+x_{4}=0, x_{5}=0\right\}$

Se había elaborado a partir de la siguiente matriz de Jordan:

$$
\left(\begin{array}{rr|rrr}
-1 & 0 & 0 & 0 & 0 \\
0 & -1 & 0 & 0 & 0 \\
\hline 0 & 0 & 2 & 0 & 0 \\
0 & 0 & 1 & 2 & 0 \\
\cline { 3 - 5 } 0 & 0 & 0 & 0 & 0
\end{array}\right)
$$




\section{Conclusiones}

La evolución de la sesión permitió repasar muchos conceptos: los que se pretendían abordar y otros preliminares, en este caso relacionados con una asignatura del mismo curso y del cuatrimestre anterior, que son imprescindibles. Los participantes mostraron gran satisfacción con la experiencia, sobre todo por la novedad en el tipo de razonamientos.

Para que tenga éxito, es necesario que el estudiante tenga unos conocimientos de la materia de estudio suficientes, por lo que no debería realizarse como actividad inicial, si no más bien de profundización posterior.

Aunque la experiencia se realizó en grupo, en un aula y guiada por el profesor. Considero que podría ser una propuesta muy interesante de trabajo individual tanto para la educación presencial como a distancia. Los estudiante podrían elaborar sus enunciados y defenderlos en un aula o bien elaborar videos explicando sus resultados. 